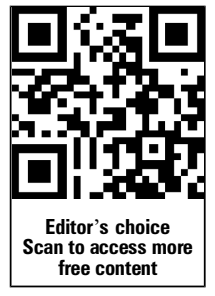

- Additional material is published online only. To view please visit the journal online (http://dx.doi.org/10.1136/ bjsports-2013-093281).

${ }^{1}$ Department of Sports Medicine, Aspetar Orthopaedic and Sports Medicine Hospital, Doha, Qatar

${ }^{2}$ Department of Sports

Medicine, The Hague Medical Centre, Antoniushove,

Leidschendam,

The Netherlands

${ }^{3}$ Department of Orthopaedics, Erasmus Medical Centre, Rotterdam, The Netherlands ${ }^{4}$ Department of Medicine, University of British Columbia, Vancouver, Canada

\section{Correspondence to} Dr Robert-Jan de Vos, Department of Orthopaedics, Erasmus Medical Centre, PO Box 2040, Rotterdam 3000 CA, The Netherlands; r.devos@erasmusmc.nl

Received 15 November 2013 Revised 28 January 2014 Accepted 31 January 2014 Published Online First 21 February 2014

\title{
Strong evidence against platelet-rich plasma injections for chronic lateral epicondylar tendinopathy: a systematic review
}

\author{
Robert-Jan de Vos, ${ }^{1,2,3}$ Johann Windt, ${ }^{4}$ Adam Weir $^{1}$
}

\begin{abstract}
Background Chronic lateral epicondylar tendinopathy is frequent in athletes, and platelet-rich plasma (PRP) is being used increasingly in its treatment.

Objective To systematically review the literature on the efficacy of PRP injections for chronic lateral epicondylar tendinopathy.

Methods The databases of PubMed, EMBASE, CINAHL, Medline OvidSP, Scopus, Google Scholar, Web of Science and Cochrane Library were searched in October 2013. Inclusion criteria were a clinical diagnosis of chronic lateral epicondylar tendinopathy, a randomised controlled trial, an intervention with a PRP injection and the outcome measures described in terms of pain and/or function. One author screened the search results and two authors independently assessed the study quality using the Physiotherapy Evidence Database (PEDro) score. A study was considered to be of high quality if its PEDro score was $\geq 6$. A best evidence synthesis was used to identify the level of evidence. Results 6 studies were included, of which four were considered to be of high quality. Three high-quality studies $(75 \%)$ and two low-quality studies showed no significant benefit at the final follow-up measurement or predefined primary outcome score when compared with a control group. One high-quality study (25\%) showed a beneficial effect of a PRP injection when compared with a corticosteroid injection (corticosteroid injections are harmful in tendinopathy). Based on the best evidence synthesis, there is strong evidence that PRP injections are not efficacious in chronic lateral epicondylar tendinopathy.
\end{abstract}

Conclusions There is strong evidence that PRP injections are not efficacious in the management of chronic lateral elbow tendinopathy.

\section{INTRODUCTION}

Chronic painful tendon disorders are frequent in athletic and inactive individuals ${ }^{1}$ and are common in general and sports medicine practice. ${ }^{2}$ They frequently lead to a decrease in activity levels in normal daily living and sometimes to cessation of sporting activities. ${ }^{1}$ It is estimated that half of all tennis players will experience some degree of lateral elbow pain during their career. ${ }^{3}$ This is commonly caused by overuse of the common extensor origin, which is frequently referred to as 'tennis elbow', though in long-standing cases we prefer the term chronic lateral epicondylar tendinopathy. ${ }^{3} 4$ The most remarkable histological sign of chronic lateral epicondylar tendinopathy is degeneration of the tendon tissue and not inflammation. ${ }^{3}$ However, the diagnosis is based on clinical examination and it can be established when there is tenderness over the common extensor origin and painful resistance against dorsiflexion of the wrist. ${ }^{4}$

Chronic lateral epicondylar tendinopathy can be resistant to traditional conservative treatment options and to improve outcomes of conservative strategies and reduce the need for surgery, more effective conservative therapies are needed. Injections with platelet-rich plasma (PRP) are said to result in tissue regeneration and have gained popularity recently. ${ }^{5}$ Platelets can be isolated from the patients' whole blood using cell-separating systems. ${ }^{6}$ The $\alpha$-granules in the platelets release many different growth factors. Previous case series showed promising results, and therefore injections with PRP are used increasingly in clinical practice. ${ }^{7-9} \mathrm{~A}$ recent narrative review concluded that the evidence in support of PRP seems to be strongest for refractory cases of lateral elbow tendinopathy. ${ }^{10}$

This systematic review aimed to examine the current literature on the efficacy of PRP injections as a treatment for chronic lateral epicondylar tendinopathy. Based on the above described positive effects of PRP, we hypothesised that there was strong evidence that PRP injections would result in a larger improvement of pain and/or function scores in patients with chronic lateral epicondylar tendinopathy than placebo or other treatment options.

\section{METHODS}

\section{Literature search}

The databases of PubMed, EMBASE, CINAHL, Medline OvidSP, Scopus, Google Scholar, Web of Science and Cochrane Library were searched without any time limits in October 2013. The main search terms were: elbow, tendinosis, tendinitis, tendinopathy, tendon injury, tennis elbow, tennis arm, chronic epicondylitis, thrombocyte, PRP and autologous blood. An overview of the complete electronic search is shown in online supplementary table S1. Additional citation tracking was performed by manual screening of the reference lists of the eligible studies. The literature search was assisted by a biomedical information specialist (WM Bramer).

\section{Study selection}

One reviewer (R-JdV) independently assessed all potential eligible trials identified by the search strategy. Articles met the eligibility criteria if the participants had a clinical diagnosis of chronic lateral epicondylar tendinopathy and the design was a randomised controlled trial (RCT). The intervention 
had to be a PRP injection and the outcome measures had to be described in terms of pain and/or function. The article had to be written in English. All titles and abstracts were assessed and all relevant articles were obtained. All relevant articles were read in full text by the reviewer to assess whether they met the eligibility criteria.

\section{Data extraction}

One reviewer (JW) recorded the study design, number of participants, intervention, control group(s), primary outcome measures, duration of follow-up, outcome in intervention group and in control group(s) using standardised data extraction forms. The methods of PRP preparation were recorded, with emphasis on the number of injections, use of anaesthetics, use of peppering technique or needling of the tendon and the activation of platelets (eg, with thrombin or calcium) with the aim of enabling quick growth factor release. Primary outcomes were extracted from the published articles to assess the effectiveness of the interventions.

\section{Quality assessment}

The Physiotherapy Evidence Database (PEDro) score was used to assess the study quality. ${ }^{11}$ This quality assessment tool assesses 11 domains, each consisting of specific items (see online supplementary table S2). The first statement is related to the external validity of the study and is not used to calculate the final quality score (item 1, as displayed in online supplementary table S4 between brackets). The reliability of the PEDro score was reported to be fair to good in a previous study. ${ }^{5}$ The PEDro score provides sufficient reliability to be applied in systematic reviews of physiotherapy trials, ${ }^{12}$ and it has been used in a recent high-quality systematic review on the effects of injection treatments in tendinopathy. ${ }^{13}$ A PEDro score of six points or higher is considered to represent a high quality study. ${ }^{5} 14$ These quality assessments of the included studies were used to categorise the level of evidence.

Two authors (JW and AW) independently assessed the quality of studies included using the PEDro forms. If there was a difference in opinion on an item, a consensus was reached by the two reviewers. If no consensus was reached, the independent opinion of a third reviewer (R-JdV) was decisive.

\section{Best evidence synthesis}

The heterogeneity of the data was evaluated after assessing the number of included studies and primary outcome measures. Data would be pooled and $\mathrm{I}^{2}$ statistics would be performed if there was suspected homogeneity of data. If data could not be pooled because of heterogeneity, a best evidence synthesis was carried out consisting of a qualitative analysis with five levels of evidence. $^{15} 16$

1. Strong evidence: provided by two or more studies with high quality and by generally consistent findings in all studies ( $\geq 75 \%$ of the studies reported consistent findings).

2. Moderate evidence: provided by one study with high quality and/or two or more studies with low quality and by generally consistent findings in all studies $(\geq 75 \%$ of the studies reported consistent findings).

3. Limited evidence: provided by only one study with low quality.

4. Conflicting evidence: inconsistent findings in multiple studies ( $<75 \%$ of the studies reported consistent findings).

5. No evidence: when no studies could be found.

\section{RESULTS}

\section{Literature search}

The initial search yielded 720 records and after removing duplicates, 318 articles were screened using the title and abstract. Thirteen studies were identified as potentially relevant, for which full text articles were retrieved. Citation tracking did not lead to any additional relevant articles. After reviewing the full text, six articles were excluded and seven articles ${ }^{17-23}$ met the inclusion criteria (figure 1).

\section{Description of included studies}

Online Supplementary table S3 presents the characteristics of the studies included. The studies of Peerbooms et $a l^{17}$ and Gosens et $a l^{18}$ used the same dataset and are therefore considered as one study. Data extraction was performed in the six studies included and a detailed description of the studies is provided below.

\section{Study design}

All six of the studies included randomly allocated participants into treatment and control groups, although only three ${ }^{17-19} 21$ reported concealing their allocation. Four of the studies reported blinding of the patients, ${ }^{17-19} 2123$ four studies reported blinding of the assessors of key outcomes ${ }^{17-20} 23$ and one study reported blinding of the individuals administering the PRP. $^{17} 18$

\section{Participants}

The median number of participants included in the studies was 80 (IQR 29.5-170). The mean patient age ranged from 36 to 51 years. Three studies ${ }^{17} 182123$ required at least 6 months of symptoms for inclusion, two studies required 3 months of symptoms, ${ }^{19}{ }^{20}$ and one required current diagnosis of tennis elbow

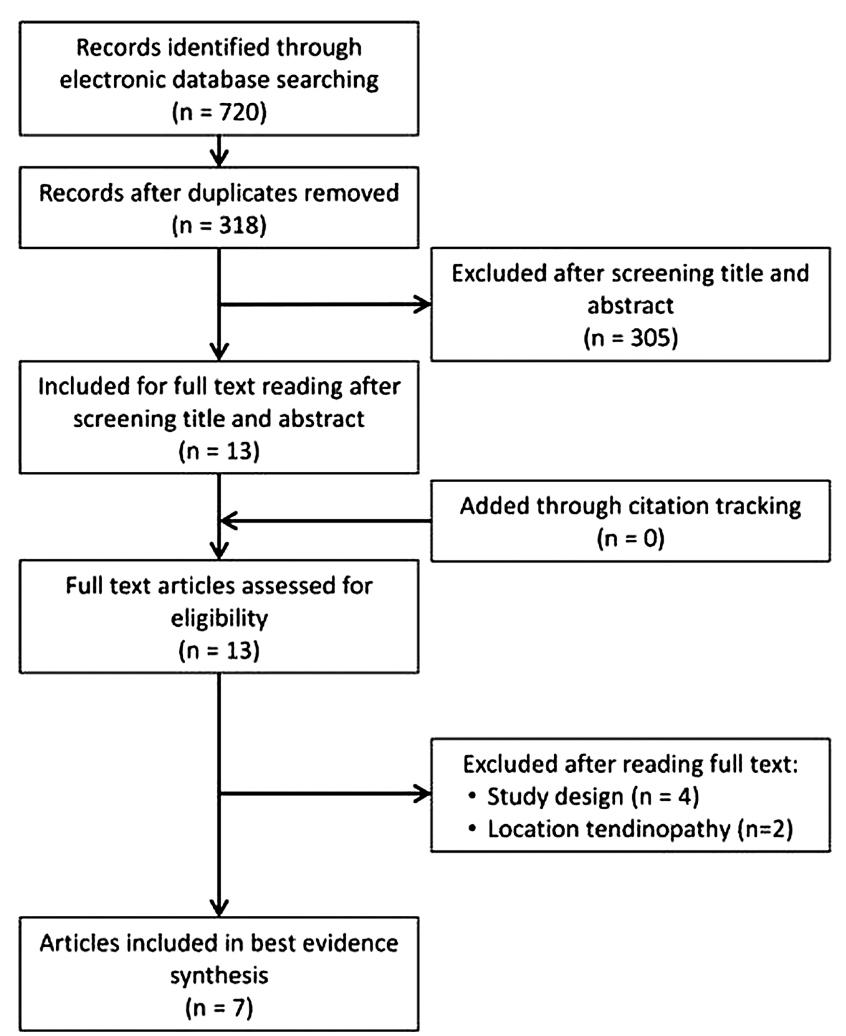

Figure 1 Study selection flow diagram. 
without any reported symptom duration. ${ }^{22}$ None of the studies included reported physical activity levels of participants' preintervention or postintervention.

\section{Interventions}

Although all studies examined PRP, the exact method and composition varied between studies. Five studies used a single injection of PRP, consisting of $2-3 \mathrm{~mL}$ of PRP with an unreported volume of bupivacaine, ${ }^{23} 3 \mathrm{~mL}$ PRP with bupivacaine and epinephrine, ${ }^{17} 183 \mathrm{~mL}$ of PRP alone, ${ }^{20} 3-3.5 \mathrm{~mL}$ of PRP alone ${ }^{19}$ and an unreported volume of PRP. ${ }^{22}$ One study used two separate injections at 1 month intervals each consisting of $1.5 \mathrm{~mL}$ PRP with $2 \mathrm{~mL}$ bupivacaine. ${ }^{21}$ Platelet count was reported in three studies, with PRP levels showing increases of at least 2 times, ${ }^{22} 2.8$ times $^{21}$ and 5.5 times $^{20}$ that of whole blood levels. One article reported that their method of preparation previously showed an average of an eightfold increase in platelet concentration. ${ }^{19}$ Two studies did not report platelet count analysis. ${ }^{17} 1823$ Leucocyte concentration was reported in one study, with an average platelet : leucocyte ratio of $111: 1{ }^{20}$ The remaining five studies did not report leucocyte concentration. Specific growth factor concentrations were reported in one study, referencing previous data on their chosen method of PRP preparation. ${ }^{20}$ For administration of the PRP, four studies used a peppering technique, ${ }^{17-20} 23$ one did not $^{21}$ and one did not report the administration technique. ${ }^{22}$ Three trials explicitly denied any activation of PRP, ${ }^{17} 182021$ while the other three did not report anything on PRP activation. ${ }^{19} 2223$

Additional treatments of stretching and strengthening exercises were reported in three of the studies. ${ }^{17-20}$ One study ${ }^{19}$ used a standard tennis elbow stretching and training programme from http://www.sportnetdoc.com; another ${ }^{17}{ }^{18}$ prescribed a physiotherapist-supervised stretching protocol for 2 weeks following the procedure, then introduced a formal eccentric strengthening programme, and one $\mathrm{e}^{20}$ had an unspecified programme of stretching and eccentric loading to be completed twice daily for 5 weeks.

Control groups varied between studies. Two studies used autologous whole blood, one using two injections combined with bupivacaine ${ }^{21}$ and one a single $3 \mathrm{~mL}$ injection of autologous peripheral whole blood. ${ }^{20}$ Corticosteroids were used as a control group in three studies: one in an unreported amount, ${ }^{22}$ one with $5 \mathrm{~mL}$ combined with bupivacaine and epinephrine ${ }^{17} 18$ and the last with $1 \mathrm{~mL}$ triamcinolone and $2 \mathrm{~mL}$ lidocaine. ${ }^{19}$ Krogh et $a l^{19}$ also used a second control group with an injection of $3 \mathrm{~mL}$ saline. One study used needling with $2-3 \mathrm{~mL}$ of bupivacaine as its control group. ${ }^{23}$

\section{Primary outcome measures}

Four studies ${ }^{17} 18 \quad 2022 \quad 23$ included visual analogue scores (VAS) as a primary outcome measure to determine pain. To evaluate function and disability, two studies also used Disabilities of the Arm, Shoulder, and Hand (DASH) scores, ${ }^{17} 1822$ and one study used the Liverpool elbow score. ${ }^{20}$ The remaining two studies evaluated outcomes using the Patient Rated Tennis Elbow Evaluation (PRTEE) questionnaire. ${ }^{19} 21$ Outcome success was determined differently in each study, reported as $25 \%$ reduction in VAS after 3 months, ${ }^{23} 25 \%$ improvement in VAS or DASH score after 2 years, ${ }^{18}$ a 25 -point improvement on the PRTEE score after 6 months $^{21}$ or improvement in PRTEE scores after 3 months. ${ }^{19}$ A single VAS at 6 weeks follow-up was used in one study. ${ }^{22}$ Follow-up duration ranged from 6 weeks to 2 years, but only two trials lasted beyond 6 months, ${ }^{17-19}$ although one of these had large dropout rates after their 3-month follow-up period. ${ }^{19}$ All but one study measured outcomes at multiple time points. $^{22}$

\section{Outcomes}

All the intervention groups reported a significant improvement in the primary outcome scores with the mean improvement being 59.6\% (SD 15.9, range 32-74). Improvement was measured as the percentage of patients with a predefined successful outcome or as a fraction of the improvement compared with the baseline measure. The outcomes in the control groups also improved significantly in all the studies with a mean improvement of $50.3 \%$ (SD 18.4, range 23-66). One of the studies included showed a beneficial effect on the primary outcome after a PRP injection when compared with a control group of corticosteroid treatment. ${ }^{17} 18$ In five studies, there was no significant effect of PRP on outcomes at predetermined follow-up times when compared with corticosteroids, ${ }^{19} 22$ autologous whole blood, ${ }^{2021}$ saline $^{19}$ or needling with bupivacaine. ${ }^{23}$

\section{Sample size calculation}

Five of the trials reported a sample size calculation. Peerbooms et $a l^{17}$ and Gosens et $a l^{18}$ reported that 42 patients were necessary per group to detect $25 \%$ reduction in VAS, although they included 50 to adjust for those lost to follow-up. Creaney et $a l^{21}$ calculated that 44 patients in each arm were required to detect a 10-point difference between groups on the PRTEE scale, and aimed to enrol 52 patients to allow for a $20 \%$ loss to follow-up. However, 150 patients were finally included. Thanasas et $a l^{20}$ reported 13 patients in each arm as its minimum sample requirement with power set at $80 \%$. They finally included 14 patients in each arm. Krogh et al ${ }^{19}$ reported sample size calculations based on the anticipated 12-month follow-up, although later adjusting to 3 months due to the large dropout rates. They had a required sample size of 17 patients in each arm to detect a mean change in the PRTEE pain score of 10 , with power at $80 \%$, eventually enrolling 20 in each arm. Finally, with power at $80 \%$, to detect a difference in success rates of $20 \%$ between groups, Mishra et $a l^{23}$ reported a required sample size of 115 in each arm. One study did not report using a sample size calculation. ${ }^{22}$

In summary, although all studies used outcome measures running from 0 to 100 , the sample size calculation ranged from 13 to 115 patients needed in one treatment arm to detect a clinically meaningful difference with enough study power.

\section{Quality assessment}

Four studies ${ }^{17} 18202223$ included VAS as the primary outcome measure. However, two of these studies dichotomised the VAS. The remaining studies used different functional outcome scores. Owing to this heterogeneity of the outcome measures and methodological quality, it was not possible to do statistical pooling of the data.

The scores on the potential risk of bias items of the studies included are shown in online supplementary table S4. There was initial disagreement between the two reviewers in 14 of the 66 items (21\%) and the opinion of the third reviewer was needed in three cases. In four of the six studies, this resulted in a different final PEDro score and in two of the six included studies, this resulted in a difference between low and high quality.

The scores ranged from 3 to 10, with an average of 6.7 (SD 2.6). Four studies were considered to be of high quality (PEDro score $\geq 6)^{17-21}$ and two studies were found to be of low quality (PEDro score $<6) .2223$ 


\section{Level of evidence}

Until now, three high-quality studies ${ }^{19-21}$ and two low-quality studies $^{22} 23$ showed no significant improvement at the final follow-up measurement or predefined primary outcome score when compared with a control group. One high-quality study ${ }^{17}$ ${ }^{18}$ showed a beneficial effect of a PRP injection for the management of chronic lateral epicondylar tendinopathy when compared with treatment with a corticosteroid injection.

As such, there is strong evidence that a PRP injection does not improve pain and/or function in chronic lateral epicondylar tendinopathy when compared with other treatment options.

\section{DISCUSSION}

Six studies were suitable for inclusion in this systematic review on the use of PRP in the treatment of chronic lateral elbow tendinopathy. Four of these studies were assessed to be of high quality. Three $(75 \%)$ of these high-quality studies showed PRP injections to be not efficacious in the treatment of chronic lateral epicondylar tendinopathy and one $(25 \%)$ demonstrated efficacy. This means that there is currently strong evidence that PRP injections are not efficacious for chronic lateral epicondylar tendinopathy.

\section{Clinical relevance of these findings}

These findings are clinically relevant because the popularity of PRP treatment is still growing, probably as a result of marketing, positive reports in laboratory studies, clinical case series and narrative reviews. ${ }^{5} 10 \mathrm{PRP}$ has been shown to have the ability to change the collagen tissue turnover and vascularisation in laboratory studies and animal studies. ${ }^{24} 25$ However, a suitable experimental model for tendinopathy in laboratory and animal studies is currently lacking due to the unknown pathophysiology. ${ }^{26}$ These initial studies were encouraging enough for the pioneers to commence using PRP for tendon injuries. Early reports in the form of case series showed an improvement in symptoms after a PRP injection. ${ }^{72}$ It should also be noted that from a previous report it is known that improvements can also be observed with a wait-and-see policy in patients with chronic lateral elbow tendinopathy. ${ }^{28}$ Randomisation of patients to a proper control group is therefore an important item in this field of research. Our systematic review shows that there is no larger improvement after PRP administration compared with other treatments or a placebo.

\section{Methodological limitations of studies included}

This systematic review shows that there are an increasing number of high-quality studies in this field. Only 3 years ago, no RCT could be included regarding PRP treatment in common tendinopathies. ${ }^{5}$ Despite the majority of the studies being scored as being of high quality, we found frequent methodological limitations. The most common were the reporting of the blinding procedure of the therapist who administered the PRP injection, the description of the allocation concealment and an adequate 'intention-to-treat principle'. These methodological limitations could potentially lead to bias. An unblinded treating physician can, consciously or unconsciously, influence the patient with knowledge of the treatment assignment. Consequently, patients may respond differently if they are aware of the treatment allocation. The allocation concealment ensures that assessors, treating physicians and patients are not able to influence the assignment of treatment to patients. If a study fails on this, the benefits of the randomisation process may be limited. The intention-to-treat principle' presumes that all patients are analysed in the groups to which they were originally allocated. Strict 'intention-to-treat analysis' is difficult to achieve because of non-compliance of the patients and loss to follow-up. It is generally accepted that a few missing outcomes will not cause a problem, but it can result in decreased study power and the introduction of bias if the loss to follow-up is related to the response to treatment. ${ }^{29}$ The PEDro score assumes that assessors are blinded if the patients are blinded if self-reported outcome measurements are used. ${ }^{11} \mathrm{We}$ feel that this is not actually correct, as the treatment allocation may have been noted somewhere, making the assessor aware of it. If there is interaction between the patient and the assessor at some stage in the study, the blinding might be at risk.

\section{Limitations of quality assessment}

There were some other items that were not assessed with the PEDro score, but which may have had a large influence on the outcome of the study. The outcome measures used were frequently not disease-specific and validated questionnaires for patients with chronic lateral elbow tendinopathy. This can enhance the quality of measurements and makes comparisons between studies easier. Another important item to address is the minimally important clinical difference. Some of the included studies reported a sample size calculation with the use of a clinically relevant difference. It is important that sample size calculations are based on predefined clinically relevant between-group differences, rather than attempting to achieve statistically significant differences. ${ }^{5}$ There was a very large difference in sample sizes between the included studies (range of 13-115 patients per treatment arm). This is very hard to explain if a standard power of $80 \%$ and standardised outcome measures running from 0 to 100 are used. Three studies even included more patients than were needed according to their power analysis. ${ }^{19-21}$ This endangers the relevance of the findings, as larger samples can lead to statistically significant but not clinically relevant differences. There might also be ethical issues because more patients are subject to a research intervention than needed.

\section{Selecting a proper control group}

A very important item in this systematic review, which could not be detected with the PEDro score, is the choice of a proper control group. The study of Peerbooms et $a l^{17}$ used a corticosteroid injection as a control group. Two high-quality reviews on treatment effects in chronic tendinopathies showed that corticosteroids provide inferior clinical outcomes (ie, harmful) compared with a wait-and-see policy after 6 months follow-up. ${ }^{13} 28$ This result was reproduced recently in a high-quality doubleblind RCT. ${ }^{30}$ This makes it impossible to answer the question whether the between-group difference observed in the study of Peerbooms et $a l^{17}$ is due to the beneficial effect of PRP or the detrimental effect of corticosteroids. Certainly, this article cannot be used to suggest that PRP is helpful as a 'naïve' (first) treatment for chronic lateral elbow tendinopathy. The same accounts for the use of anaesthetics as a control group, which was used in three studies (two in combination with corticosteroids). From a recent animal study, it is known that bupivacaine has detrimental effects on tendon cell viability, which may put the tendon at increased risk of overload or acute injury. ${ }^{31}$ This stresses the need for placebo-controlled studies in the field of treatments for tendinopathies. ${ }^{32}$ Only one study included in our review ${ }^{19}$ used a saline placebo injection in the control group.

\section{Limitations of this systematic review}

There are a few limitations of this systematic review. The first limitation of our study is that we analysed the results of the 
predefined outcome measures or the final follow-up measurements if there was no predefined primary outcome. One study showed a beneficial effect of PRP on the VAS scale compared with whole autologous blood after 6 weeks. ${ }^{20}$ However, at the same follow-up time, there was no difference in a functional outcome measure. In one study, the follow-up time was extended for unknown reasons. ${ }^{23}$ These authors did not show a beneficial effect of PRP compared with a bupivacaine injection at their predefined outcome moment after 12 weeks, but a beneficial effect of PRP was reported after 24 weeks follow-up. However, there was an extremely high loss to follow-up of patients at this time point $(52 \%)$, which increases the risk of attrition bias. The second limitation of our systematic review is the high number of disagreements between the authors in the quality assessment. There are two main reasons for this result. First, the quality of the writing of the articles was very disappointing in some cases, and most authors seem not to have followed the CONSORT guidelines when reporting their methods or results. This makes finding the methods and interpreting them challenging based on the level of description in the articles. In one study, ${ }^{21}$ disagreement was found in 5 of 11 items. Authors should report their study results according to the CONSORT statement ${ }^{29}$ to improve the quality of the reporting of their study. Failure to report in this way increases disagreement as reviewers are forced to rely on implicit claims on certain items rather than clearly stated procedures. Second, a few items on the PEDro assessment form are not optimally described. As mentioned above, the PEDro form assumes assessors to be blinded when patients are blinded if self-reported outcome measurements are used. We feel that this is not necessarily the case. The PEDro form does not examine the statistics used. Many studies assessed the outcome at multiple moments and some of them did not use the correct techniques for examining this. The CONSORT statement advises correcting for baseline factors that may influence outcome where possible. ${ }^{29}$ The PEDro form does not capture this. We found in practice that scoring of the intention-to-treat analysis was difficult as authors wrote that they had performed the intention-to-treat analysis but at no point described how they handled this in their statistical methods or, as in the case of Mishra et al, ${ }^{23}$ used last value carried forward imputation, which carries a high risk of bias, especially with more than $20 \%$ attrition rates. ${ }^{33} 34$ Some items will always be hard to quantify using an assessment form. For example, we consistently evaluated one study ${ }^{19}$ as a high-quality study. However, there might be subtle changes in the handling of outcome measures or preparation of PRP that can potentially negatively influence the outcome. ${ }^{35}$ These elements would not be detected with the PEDro score. A third limitation of this systematic review is the fact that we were not able to pool data and perform a quantitative analysis due to the heterogeneity of the data. We aimed to provide a quantitative analysis using the calculated percentages of improvement in pain and/or function scores. This percentage improvement is a suboptimal measure because it is dependent on the baseline score. However, it was the best available measure we could apply, and furthermore, due to the randomisation, the baseline values are evenly distributed in all treatment arms and are therefore do not affect the comparative results. Furthermore, an advantage of a qualitative analysis is that all included studies are evaluated in the final analysis, resulting in a level of evidence based on all included studies. In quantitative analysis, high-quality studies are sometimes excluded from the final analysis because of the outcome measure. If for instance a disease-specific validated outcome measure is used but pooling is performed with a less-specific and less-relevant VAS, bias could be introduced. ${ }^{36}$ Such bias is not present in a qualitative analysis.

\section{Recommendations for future studies}

We recommend that future studies in this field should use appropriate control groups with blinded saline injections. Authors should follow the CONSORT guidelines when reporting their studies to allow for better evaluation of the quality.

\section{CONCLUSION}

There is currently strong evidence that PRP injections are not efficacious in the treatment of chronic lateral epicondylar tendinopathy. Three high-quality studies showed no significant benefit of PRP at the final follow-up measurement or predefined primary outcome score when compared with a control group. The only high-quality study that showed a positive effect of PRP compared it with a detrimental corticosteroid injection.

\section{What are the new findings?}

- This systematic review shows strong evidence that platelet-rich plasma (PRP) injections are not efficacious in the treatment of chronic lateral epicondylar tendinopathy.

- The most common methodological limitations were the reporting of the blinding procedure of the therapist who administered the PRP injection, the description of the allocation concealment and an adequate 'intention-to-treat principle'.

- Future studies in this field should use appropriate control groups with saline injections.

Acknowledgements The authors would like to thank WM Bramer, biomedical information specialist in the Erasmus University medical centre, Rotterdam, the Netherlands, for assistance in the search strategy.

Contributors R-JdV contributed to the search strategy, data summary, analysis and interpretation of the data and made the first draft of the manuscript. JW analysed and interpreted the data, performed the data extraction and the revision of the manuscript. AW analysed and interpreted the data and revised the manuscript. All authors gave final approval for the version to be published.

Competing interests None.

Provenance and peer review Not commissioned; externally peer reviewed.

\section{REFERENCES}

1 Maffulli N, Wong J, Almekinders LC. Types and epidemiology of tendinopathy. Clin Sports Med 2003;22:675-92.

2 De Jonge $S$, van den Berg C, de Vos RJ, et al. Incidence of midportion Achilles tendinopathy in the general population. Br J Sports Med 2011;45:1026-8.

3 Tosti R, Jennings J, Sewards JM. Lateral epicondylitis of the elbow. Am J Med 2013;126:357.e1-357.e6.

4 De Smedt T, de Jong A, Van Leemput W, et al. Lateral epicondylitis in tennis: update on aetiology, biomechanics and treatment. Br J Sports Med 2007;41:816-19.

5 De Vos RJ, van Veldhoven PL, Moen MH, et al. Autologous growth factor injections in chronic tendinopathy: a systematic review. $\mathrm{Br}$ Med Bull 2010;95:63-77

6 Sampson S, Gerhardt M, Mandelbaum B. Platelet rich plasma injection grafts for musculoskeletal injuries: a review. Curr Rev Musculoskelet Med 2008;1:165-74.

7 Mishra A, Pavelko T. Treatment of chronic elbow tendinosis with buffered platelet-rich plasma. Am J Sports Med 2006;34:1774-8.

8 Edwards SG, Calandruccio JH. Autologous blood injections for refractory lateral epicondylitis. J Hand Surg Am 2003;28:272-8.

9 Connell DA, Ali KE, Ahmad M, et al. Ultrasound-guided autologous blood injection for tennis elbow. Skeletal Radiol 2006:35:371-7. 
10 Hall MP, Ward JP, Cardone DA. Platelet rich placebo? Evidence for platelet rich plasma in the treatment of tendinopathy and augmentation of tendon repair. Bull Hosp Jt Dis 2013;71:54-9.

11 Maher CG, Sherrington C, Herbert RD, et al. Reliability of the PEDro scale for rating quality of randomized controlled trials. Phys Ther 2003:83:713-21.

12 Moher D, Cook DJ, Eastwood S, et al. Improving the quality of reports of meta-analyses of randomised controlled trials: the QUOROM statement. Quality of Reporting of Meta-analyses. Lancet 1999;354:1896-900.

13 Coombes BK, Bisset L, Vicenzino B. Efficacy and safety of corticosteroid injections and other injections for management of tendinopathy: a systematic review of randomised controlled trials. Lancet 2010;376:1751-67.

14 Woodley BL, Newsham-West RJ, Baxter GD. Chronic tendinopathy: effectiveness of eccentric exercise. Br J Sports Med 2007;41:188-98; discussion 199.

15 Van Tulder M, Furlan A, Bombardier C, et al. Updated method guidelines for systematic reviews in the Cochrane collaboration back review group. Spine 2003;28:1290-9.

16 Swart NM, van Linschoten R, Bierma-Zeinstra SMA, et al. The additional effect of orthotic devices on exercise therapy for patients with patellofemoral pain syndrome: a systematic review. Br J Sports Med 2012;46:570-7.

17 Peerbooms JC, Sluimer J, Bruijn DJ, et al. Positive effect of an autologous platelet concentrate in lateral epicondylitis in a double-blind randomized controlled trial: platelet-rich plasma versus corticosteroid injection with a 1-year follow-up. Am J Sports Med 2010;38:255-62.

18 Gosens T, Peerbooms JC, van Laar W, et al. Ongoing positive effect of platelet-rich plasma versus corticosteroid injection in lateral epicondylitis: a double-blind randomized controlled trial with 2-year follow-up. Am J Sports Med 2011;39:1200-8

19 Krogh TP, Fredberg U, Stengaard-Pedersen K, et al. Treatment of latera epicondylitis with platelet-rich plasma, glucocorticoid, or saline: a randomized, double-blind, placebo-controlled trial. Am J Sports Med 2013:41:625-35.

20 Thanasas C, Papadimitriou G, Charalambidis C, et al. Platelet-rich plasma versus autologous whole blood for the treatment of chronic lateral elbow epicondylitis: a randomized controlled clinical trial. Am J Sports Med 2011:39:2130-4.

21 Creaney L, Wallace A, Curtis M, et al. Growth factor-based therapies provide additional benefit beyond physical therapy in resistant elbow tendinopathy: a prospective, single-blind, randomised trial of autologous blood injections versus platelet-rich plasma injections. Br J Sports Med 2011;45:966-71.
22 Omar AS, Ibrahim ME, Ahmed AS, et al. Local injection of autologous platelet rich plasma and corticosteroid in treatment of lateral epicondylitis and plantar fasciitis: randomized clinical trial. Egypt Rheumatol 2012;34:43-9.

23 Mishra AK, Skrepnik NV, Edwards SG, et al. Platelet-rich plasma significantly improves clinical outcomes in patients with chronic tennis elbow: a double-blind, prospective, multicenter, controlled trial of 230 patients. Am J Sports Med 2014;42:463-71

24 De Mos M, van der Windt AE, Jahr $\mathrm{H}$, et al. Can platelet-rich plasma enhance tendon repair? A cell culture study. Am J Sports Med 2008;36:1171-8.

25 Bosch G, van Schie HTM, de Groot MW, et al. Effects of platelet-rich plasma on the quality of repair of mechanically induced core lesions in equine superficial digital flexor tendons: a placebo-controlled experimental study. J Orthop Res 2010;28:211-17

26 Warden SJ. Development and use of animal models to advance tendinopathy research. Front Biosci (Landmark Ed) 2009;14:4588-97.

27 Hechtman KS, Uribe JW, Botto-vanDemden A, et al. Platelet-rich plasma injection reduces pain in patients with recalcitrant epicondylitis. Orthopedics 2011;34:92.

28 Smidt N, van der Windt DAWM, Assendelft WJ, et al. Corticosteroid injections, physiotherapy, or a wait-and-see policy for lateral epicondylitis: a randomised controlled trial. Lancet 2002;359:657-62.

29 Schulz KF, Altman DG, Moher D, et al. CONSORT 2010 statement: updated guidelines for reporting parallel group randomized trials. Ann Intern Med 2010;152:726-32.

30 Coombes BK, Bisset L, Brooks P, et al. Effect of corticosteroid injection, physiotherapy, or both on clinical outcomes in patients with unilateral lateral epicondylalgia: a randomized controlled trial. JAMA 2013;309:461-9.

31 Lehner C, Gehwolf R, Hirzinger C, et al. Bupivacaine induces short-term alterations and impairment in rat tendons. Am J Sports Med 2013;41:1411-18.

32 De Vos RJ, Weir A, van Schie HTM, et al. Platelet-rich plasma injection for chronic Achilles tendinopathy: a randomized controlled trial. JAMA 2010;303:144-9.

33 Dumville JC, Torgerson DJ, Hewitt CE. Reporting attrition in randomised controlled trials. BMJ 2006:332:969-71.

34 Aspenberg P, Ranstam J, O'Sullivan K, et al. Platelet-rich plasma for chronic tennis elbow: letters to the editor. Am J Sports Med 2014:42:NP1-6.

35 Wilson JJ, Rabago DP, Lee KS, et al. Platelet-rich plasma treatment for lateral epicondylitis: letter to the editor. Am J Sports Med 2013;41:NP33-5.

36 Moraes VY, Lenza M, Tamaoki MJ, et al. Platelet-rich therapies for musculoskeletal soft tissue injuries. Cochrane Database Syst Rev 2013;12:CD010071. 